\title{
Reference Tracking for Linear Time Invariant Systems with a Relay Control *
}

\author{
Zohra Kader ${ }^{1}$ and Antoine Girard ${ }^{1}$
}

\begin{abstract}
This paper investigates the problem of tracking a reference trajectory for Linear Time Invariant (LTI) systems using a relay feedback. First of all, the problem of a reference tracking is reformulated as a stabilization problem of the tracking error. Then, under some conditions on the reference inputs a design approach of a relay feedback controller that guarantees a perfect tracking of the reference trajectory is provided. Moreover, conditions in LMI form are proposed in order to synthesize a relay feedback and provide an estimation of the domain of attraction. Secondly, the case where the reference inputs can take the same values as the control input is considered. In this context, achieving a perfect tracking is no longer possible. Thus, using the comparison principle, a relay feedback controller that guarantees a practical stabilization of the tracking error is proposed. An estimation of the domain of attraction along with the bound of the tracking error are equally provided. Finally, computer simulations show the efficiency of the developed method over a numerical example.
\end{abstract}

\section{INTRODUCTION}

Relay feedback controllers are omnipresent in different application fields [8], [10], [20], [21]. They are known to be simple, robust, and able to emulate locally the behavior of a linear static feedback [6]. These properties make them an interesting substitute to continuous controllers - see for instance [8], [9]. Despite these advantages, relay feedback controllers have been shown to exhibit some difficulties and undesirable phenomena [11], [12]. Indeed, it has been shown in the literature that relay feedback controllers present sliding modes, chattering and limit cycles. The presence of these complex behaviors make their study very challenging. However, these phenomena must not be neglected and should be considered in the design procedures. In particular, for systems with sliding modes the notion of system's solution must be reviewed in order to take into account the dynamics obtained by fast switching [5], [7]. Up to now, the investigations on relay feedback controllers design are limited to the stabilization problem to an equilibrium point. Frequency domain methods [3] and LMI approaches [16], [17] have been used for this purpose.

Here, we are interested on designing relay feedback controller that enforces the trajectories of an LTI system to track a reference trajectory. The proposed approach is inspired from the results in [9] and [13]. First, we reformulate the

\footnotetext{
* This work has received funding from the European Research Council (ERC) under the European Union's Horizon 2020 research and innovation program (grant agreement No 725144).

Zohra Kader $^{1}$ and Antoine Girard ${ }^{1}$ are with Laboratoire des Signaux et Systèmes (L2S) CNRS, CentraleSupélec, Université Paris-Sud, Université Paris-Saclay 3, rue JoliotCurie, 91192 Gif-sur-Yvette, France. \{zohra.kader , antoine.girard\}@l2s.centralesupelec.fr.
}

problem of a reference tracking as a stabilization problem of the tracking error. Then, under some conditions on the reference inputs a design approach of a relay feedback controller that guarantees a perfect tracking of the reference trajectory together with an estimation of the domain of attraction is provided. Moreover, an LMI approach that allows the design of a stabilizing relay feedback while providing an estimation of the domain of attraction is proposed. Secondly, the case where the reference inputs can take the same values as the relay feedback is considered. In this context, using the comparison principle, the designed relay feedback guarantees a practical stabilization of the tracking error. An estimation of the domain of attraction and the bound of the tracking error are equally provided. Finally, computer simulations are performed in order to show the efficiency of the developed method.

The paper is organized as follows: Section II presents the system description, the stability notions, and the problem under study. Under some restrictions on the reference inputs, a design approach of a relay feedback controller that ensures the perfect tracking of the reference trajectory is provided in Section III. In addition, a constructive method based on LMIs is proposed in the same section. In Section IV, using the comparison principle a relay feedback controller that ensures the boundedness of the closed-loop system trajectories is provided. A numerical example is given to illustrate the efficiency and the limits of the presented method. Finally, concluding remarks are provided in the last section.

\section{A. Notations}

In this paper we use the notation $\mathbb{R}^{+}$to refer to the interval $[0, \infty)$. The transpose of a matrix $M$ is denoted by $M^{T}$ and if the matrix is symmetric the symmetric elements are denoted by $*$. The notation $M \succeq 0$ (resp. $M \preceq 0$ ) means that the matrix $M$ is positive (resp. negative) semi-definite, and the notation $M \succ 0$ (resp. $M \prec 0$ ) means that it is positive definite (resp. negative definite). $M_{(i)}$ refers to the $i$-th row of a matrix (or a vector).

The identity matrix is denoted by $I$ and both notations $\operatorname{eig}_{\min }(M)$ and $\operatorname{eig}_{\max }(M)$ are used to refer to the minimum and maximum eigenvalue respectively of a matrix $M$. For a positive definite matrix $M \in \mathbb{R}^{n \times n}$ and a positive scalar $\gamma$, we denote by $\mathcal{E}(M, \gamma)$ the ellipsoid

$$
\mathcal{E}(M, \gamma)=\left\{x \in \mathbb{R}^{n}: x^{T} M x \leq \gamma\right\}
$$

and for all positive scalar $r$, we denote by $\mathcal{B}(0, r)$ the ball

$$
\mathcal{B}(0, r)=\mathcal{E}(I, r)=\left\{x \in \mathbb{R}^{n}:\|x\| \leq r\right\},
$$


where $\|$.$\| is used to denote the Euclidean norm for a vector$ and the associated norm for a matrix. For a given set $\mathcal{S}$, the notation $\operatorname{Conv}\{\mathcal{S}\}$ indicates the convex hull of the set, int $\{\mathcal{S}\}$ its interior and $\mathcal{S}$ its closure and finally the closed convex hull of the set $\mathcal{S}$ is denoted by $\overline{\operatorname{Conv}}\{\mathcal{S}\}$. The minimum argument of a given function $f: \mathcal{S} \longrightarrow \mathbb{R}$ such that the set $\mathcal{S} \subset \mathbb{R}$ is a finite set of vectors is denoted by

$$
\arg \min f=\{y \in \mathcal{S}: f(y) \leq f(z), \forall z \in \mathcal{S}\} .
$$

For a positive integer $N$, we denote by $\mathcal{I}_{N}$ the set $\{1, \ldots, N\}$. By $\psi_{N}$ we denote the unit simplex

$\psi_{N}=\left\{\alpha=\left(\alpha_{1}, \ldots, \alpha_{N}\right)^{T} \in \mathbb{R}^{n}: \sum_{i=1}^{N} \alpha_{i}=1, \alpha_{i} \geq 0, i \in \mathcal{I}_{N}\right\}$.

\section{SYSTEM DESCRIPTION AND PROBLEM STATEMENT}

\section{A. System description}

Consider the linear system

$$
\dot{x}=A x+B u,
$$

with $x \in \mathbb{R}^{n}$ and an input $u$ which takes values in the set $\mathcal{V}=\left\{v_{1}, \ldots, v_{N}\right\} \subset \mathbb{R}^{m} . A \in \mathbb{R}^{n \times n}$, and $B \in \mathbb{R}^{n \times m}$ are the matrices describing the system.

This paper deals with the design of a relay feedback controller that enforces the system state to asymptotically track the reference signal $x_{r}$ which is given by the reference model

$$
\dot{x}_{r}=A x_{r}+B u_{r},
$$

where $u_{r}$ is a piece-wise constant and right continuous function from $\mathbb{R}^{+}$to the finite set of vectors $\mathcal{V}^{r}=$ $\left\{v_{1}^{r}, v_{2}^{r}, \ldots, v_{\tilde{N}}^{r}\right\}$, with a finite number of discontinuities on every bounded interval of $\mathbb{R}^{+}, A$ and $B$ are defined in (5). Note that the number of the reference input vectors $\tilde{N}>0$ can be equal to $N$.

Given the desired reference $x_{r}$ from (6) and the system model (5), the open loop tracking error system is

$$
\begin{gathered}
\dot{e}=A e+B u-B u_{r}, \\
u \in \mathcal{V}, u_{r} \in \mathcal{V}^{r}, e:=x-x_{r} .
\end{gathered}
$$

Due to the formulation (7), the tracking problem can be restated as the design of a relay feedback controller that asymptotically stabilizes the error system to the origin. To this end, in the sequel we assume that:

A-1 The pair $(A, B)$ is stabilizable, which means that there exists a matrix $K$ such that the closed-loop matrix $A_{c l}=A+B K$ is Hurwitz.

A-2 The set $\operatorname{Conv}\{\mathcal{V}\}$ is nonempty, and the null vector is contained in its interior $(0 \in \operatorname{int}\{\operatorname{Conv}\{\mathcal{V}\}\})$.

Here, we consider a controller given by

$$
u(e) \in \underset{v \in \mathcal{V}}{\arg \min } e^{T} \mathbf{S} v,
$$

where the matrix $\mathrm{S} \in \mathbb{R}^{n \times m}$ characterizes the switching hyperplanes. The formulation (8) can be interpreted as networked control systems with quantization [15]. Moreover, the formulation of the controller (8) encompasses the classical sign function in the classical relay feedback. Therefore, if $\mathcal{V}=\left\{v_{1}, v_{2}\right\}=\{-v, v\}$ with $v>0$ then we get

$$
\begin{aligned}
u(e) & =-v \operatorname{sign}\left(\mathrm{S}^{T} e\right) \\
& \in\left\{\begin{array}{l}
v \text { if } \mathrm{S}^{T} e<0, \\
\{-v, v\} \text { if } \mathrm{S}^{T} e=0, \\
v \text { if } \mathrm{S}^{T} e>0 .
\end{array}\right.
\end{aligned}
$$

The closed-loop system is modeled by a differential equation with a discontinuous right hand-side. Consequently, to study the stability of the system (7), (8) we will consider the Filippov solutions of differential inclusions. Next, we provide the definitions of differential inclusions and their solutions [1], [2], [5], and [7].

\section{B. Solution concept and stability notions}

The interconnection (7), (8) is the closed-loop system modeled by a discontinuous differential equation of the form

$$
\dot{e}=A e+B u(e)-B u_{r}=f(t, e) .
$$

Therefore, to the discontinuous closed-loop system (10) we associate the differential inclusion

$$
\dot{e} \in \mathcal{F}(t, e),
$$

with $\mathcal{F}(t, e)$ the set-valued map which can be computed from the differential equation with a discontinuous right hand-side using the construction given by :

$$
\mathcal{F}(t, e)=\bigcap_{\delta>0} \bigcap_{\mu(\mathcal{S})=0} \overline{\operatorname{Conv}}\{f(t, \mathcal{B}(e, \delta)) \backslash \mathcal{S}\}, e \in \mathbb{R}^{n}, t \in \mathbb{R}^{+},
$$

where $\overline{\mathrm{Conv}}$ is the closed convex hull, $\mathcal{B}(e, \delta)$ is the open ball centered on $e$ with radius $\delta$, and $\mathcal{S}$ is a set of measure zero with $\mu(\mathcal{S})$ its measure in the sens of Lebesgue - see for instance [2], [5], [7], [18], and the references therein. The closed-loop system is then modeled by a differential inclusion for which the notion of a solution was defined in [7], and recalled in the following.

Definition 1: (Filippov solution) Consider the closed-loop system (10) and its associated differential inclusion (11). A Filippov solution of the discontinuous systems (7), (8) over the interval $\left[t_{a}, t_{b}\right] \subset[0, \infty)$ is an absolutely continuous mapping $y:\left[t_{a}, t_{b}\right] \longrightarrow \mathbb{R}^{n}$ satisfying:

$$
\dot{y}(t) \in \mathcal{F}(t, y(t)), \quad \text { for almost all } t \in\left[t_{a}, t_{b}\right],
$$

with $\mathcal{F}(t, e)$ given by (12)

A differential inclusion has at least one solution if the set valued map $\mathcal{F}(t, e)$ is locally bounded and takes nonempty, compact and convex values [1], [2], [5], [7].

Definition 2 (Equilibrium point): $e_{e q}$ is said to be an equilibrium point of the differential inclusion (11) if $0 \in$ $\mathcal{F}\left(t, e_{e q}\right)$ for all $t \geq t_{0} \geq 0$.

Hereafter the notions of stability that we will use are introduced.

Definition 3 (Local uniform exponential stability): The equilibrium point $e_{e q}=0$ of the differential inclusion (11) is said to be locally uniformly exponentially stable with a decay rate $\alpha$, if there exist a set $\mathcal{D} \subset \mathbb{R}^{n}, 0 \in \operatorname{int}\{\mathcal{D}\}$, and 
strictly positive scalars $\kappa$ independent of $t_{0}$, and $\alpha$ such that for all Filippov solutions $e(t)$ of (11) with $e\left(t_{0}\right) \in \mathcal{D}$,

$$
\|e(t)\| \leq \kappa e x p\left(-\alpha\left(t-t_{0}\right)\right)\left\|e\left(t_{0}\right)\right\|, \forall t \geq t_{0} \geq 0 .
$$

A set $\mathcal{D}$ satisfying these properties is usually called an estimation of the domain of attraction.

We recall that sufficient conditions for the local uniform exponential stability of Filippov solutions in the case of systems modeled by differential equations with a discontinuous right hand-side $\dot{e}=f(t, e)$, are given by the existence of a strict Lyapunov function $V, V(t, 0)=0, V(t, e) \geq 0$, $\forall e \neq 0, t \geq t_{0} \geq 0$ and continuous positive definite functions $W_{1}$ and $W_{2}$ (i.e. $W_{i}(0)=0$ and $W_{i}(e)>0$ for all $e \neq 0$ and $i \in\{1,2\}$ ) (see [7], Chapter 3, page 153, Theorem 1), such that

$$
W_{1}(e) \leq V(t, e) \leq W_{2}(e)
$$

and

$$
\sup _{y \in \mathcal{F}(t, e)}\left\{\frac{\partial V}{\partial t}+\frac{\partial V}{\partial e} y\right\} \leq-2 \alpha V(t, e), \forall e \in \mathcal{D} \backslash\{0\}, \forall t \geq 0,
$$

for some domain $\mathcal{D}$ such that $0 \in \mathcal{D}$ and some positive scalar $\alpha$.

We adapt as follows the concept of practical stability from [19] to the context under study. be

Definition 4 (Practical stability): System (11) is said to

- Practically stable, if given $\left(R_{1}, R_{2}\right)$ with $0<R_{1}<R_{2}$ then $\left\|e\left(t_{0}\right)\right\|<R_{1} \Rightarrow\|e(t)\|<R_{2}, \forall t \geq t_{0} \geq 0$;

- Uniformly practically stable, if it is practically stable for all $t_{0} \geq 0$.

For more details about practical and exponential stability, one can refer to [7], [14].

\section{CASE 1. PERfECt REFERENCE TRACKING}

\section{A. Control design}

In this section, we consider the case where a perfect tracking of the reference trajectory can be achieved by a relay feedback. To this end, let us make the following additional assumption:

A-3 The set of reference inputs $\operatorname{Conv}\left\{\mathcal{V}^{r}\right\}$ is contained in the interior of the convex set $\operatorname{Conv}\{\mathcal{V}\}$ i.e., $\operatorname{Conv}\left\{\mathcal{V}^{r}\right\} \subset \operatorname{int}\{\operatorname{Conv}\{\mathcal{V}\}\}$.

Theorem 1: Assume that A.1 and A.3 hold. Then there exist a matrix S such that the closed-loop system (7), (8) is locally uniformly exponentially stable with a decay rate $\alpha$ in a domain that contains the origin.

The control design approach proposed in Theorem 1 can be used to provide a constructive method of relay feedback controllers that enforce system (5) to track the reference trajectory (6). In the next section, using a quadratic Lyapunov function we propose LMIs (Linear Matrix Inequalities) criteria for the design of relay controllers while providing an estimation of the domain of attraction.

\section{B. LMIs solution}

The result of Theorem 1 has a qualitative nature. In practice, it is useful to find a constructive procedure which provides a switching law which ensures the stability of the system (7) together with an estimation of the domain of attraction. Here, we provide a numerical approach to deal with this problem. More precisely, an LMI solution is proposed hereafter. In order to express the result note that for any set $\mathcal{V}$ there exists a finite number $n_{h}$ of vectors $h_{i} \in \mathbb{R}^{1 \times m}, i \in \mathcal{I}_{n_{h}}$ such that

$$
\operatorname{Conv}\{\mathcal{V}\}=\left\{u \in \mathbb{R}^{m}: h_{i} u \leq 1, i \in \mathcal{I}_{n_{h}}\right\}
$$

Theorem 2: Assume that A.1 and A.3 hold. Consider the linear closed-loop system (7), (8) and a tuning parameter $\delta>0$. If there exist a positive definite matrix $Q \in \mathbb{R}^{n \times n}$ and a matrix $Y \in \mathbb{R}^{m \times n}$ such that the LMIs

$$
\begin{gathered}
Q A^{T}+A Q+Y^{T} B+B Y \leq-2 \delta Q \\
{\left[\begin{array}{cc}
1 & \tilde{h}_{i, j} Y \\
Y^{T} \tilde{h}_{i, j}^{T} & Q
\end{array}\right] \geq 0, \forall i \in \mathcal{I}_{n_{h}}, \forall j \in \mathcal{I}_{\tilde{N}},}
\end{gathered}
$$

with $\tilde{h}_{i, j}=\frac{h_{i}}{1-h_{i} v_{j}^{r}}$, are feasible then the origin of system (7) with the switching law

$$
u(e) \in \underset{v \in \mathcal{V}}{\arg \min } e^{T} Q^{-1} B v,
$$

is locally uniformly exponentially stable with a decay rate $\delta$.

Remark 1: The feasibility of the set of LMIs (18)-(19) allows the design of the matrix $\mathrm{S}=M B$ that characterizes the switching hyperplane and provides an estimation $\mathcal{E}(M, 1)$ of the domain of attraction. More precisely, any solution of the closed-loop system (7), (8) starting in the domain of attraction $\mathcal{E}(M, 1)$ converges to the origin exponentially with a decay rate $\delta$. In this case, the trajectories of system (5) will converge to (or track) the trajectories of the reference system (6).

In this section, we have shown that if the reference input $u_{r}$ satisfies Assumption A.3 then we are able to design a relay controller ensuring that the tracking error will tend to zero as $t$ tends to infinity. When Assumption A.3 does not hold, only practical stabilization of the tracking error is possible. This case is considered in the next section.

\section{Case 2. Practical stabilization of the TRACKING ERROR}

Here, we are interested in the case where Assumption A.3 does not hold i.e, the reference imputs $u_{r}$ can take values in $\operatorname{Conv}\{\mathcal{V}\}$. In this case, one can only achieve practical stability of the error system (7) by the relay controller (8) i.e., one is interested on specific trajectory bounds of (7), (8). In order to provide stabilization criteria of system (7), we use the so-called comparison Lemma [14], [19]. In order to build the comparison system, we consider that Assumptions A. 1 and A. 2 hold. Therefore, thanks to the fact that the pair $(A, B)$ is stabilizable, there exists a gain $K$ such that $A_{c l}=$ 
$A+B K$ is Hurwitz. Furthermore, for all $\delta>0$ there exists a matrix $M=M^{T} \succ 0$ verifying

$$
A_{c l}^{T} M+M A_{c l} \preceq-2 \delta M .
$$

Moreover, since the set $\operatorname{Conv}\{\mathcal{V}\}$ is nonempty and the null vector is contained in its interior $(0 \in \operatorname{int}\{\operatorname{Conv}\{\mathcal{V}\}\})$, then there exists a neighbourhood of the origin $\mathcal{E}(M, \gamma)$ with $\gamma>$ 0 such that for all $e \in \mathcal{E}(M, \gamma)$ we have

$$
K e \in \operatorname{Conv}\{\mathcal{V}\} \text {. }
$$

Thus, for all $e \in \mathcal{E}(M, \gamma)$ there exist positive scalars $\alpha_{j}(e)$, $j \in \mathcal{I}_{N}$, such that $\sum_{j=1}^{N} \alpha_{j}(e)=1$ and

$$
K e=\sum_{j=1}^{N} \alpha_{j}(e) v_{j} .
$$

Now, let us make the following assumption:

A-4 There exists a function $\eta: \mathbb{R}^{+} \rightarrow \mathbb{R}$ satisfying

$$
\dot{\eta}=-\lambda \eta+\pi u_{r}^{T} u_{r}, \quad \eta\left(t_{0}\right) \geq 0,
$$

where $\pi$ is a positive real satisfying $\pi>\frac{\xi_{\max }}{2 \delta}$ with $\delta>0$ is defined in (21) and $\xi_{\max }>0$ satisfies the inequality $e^{T} M B B^{T} M e \leq \xi_{\max } e^{T} M e$ for all $e \in \mathbb{R}^{n}$, and $\lambda=2 \delta-\pi^{-1} \xi_{\max }>0$. Moreover, for all $t \geq t_{0}$, $\eta(t) \leq \varepsilon \leq \gamma$ where $\varepsilon>0$ is such that $K \mathcal{E}(M, \varepsilon) \subset$ $\operatorname{Conv}\{\mathcal{V}\}$.

The comparison system (24) is utilized in order to prove the uniform practical stability of the closed-loop system (7), (8). This result is given in the following theorem.

Theorem 3: Consider system (7), (8) under Assumptions A.1, A.2 and A.4. Then, there exist a positive scalar $\gamma$ and matrices $M=M^{T} \succ 0$ and $\mathrm{S}=M B$ such that system (7), (8) is uniformly practically stable with $R_{1}=\sqrt{\frac{\eta\left(t_{0}\right)}{e i g_{\min }(M)}}$ and $R_{2}=\sqrt{\frac{\varepsilon}{e i g_{\min }(M)}}$.

Remark 2: One may remark that:

1) the result in Theorem 3 means that the existence of a static feedback controller $\tilde{u}=K e$ and a comparison system given by Assumption A.4 implies the existence of a relay feedback that stabilizes system (7). The designed relay feedback guarantees that the closedloop system has the same stability properties as the comparison system (24).

2) the result of Theorem 3 is constructive. Indeed, if the inequality (21) is satisfied and if there exists a comparison system defined as in Assumption A.4 then the closed-loop system (7), (8) is uniformly practically stable with a switching hyperplane given by $S=M B$. Inequality (21) can be easily reformulated as an LMI design condition [4]: there exist $Q=Q^{T} \succ 0$ and a matrix $Y \in \mathbb{R}^{m \times n}$ such that

$$
Q A^{T}+A Q+Y^{T} B+B Y \leq-2 \delta Q
$$

with $Q=M^{-1}$ and $K=Y M$. Then, the switching hyperplane is characterized by $\mathrm{S}=M B$.

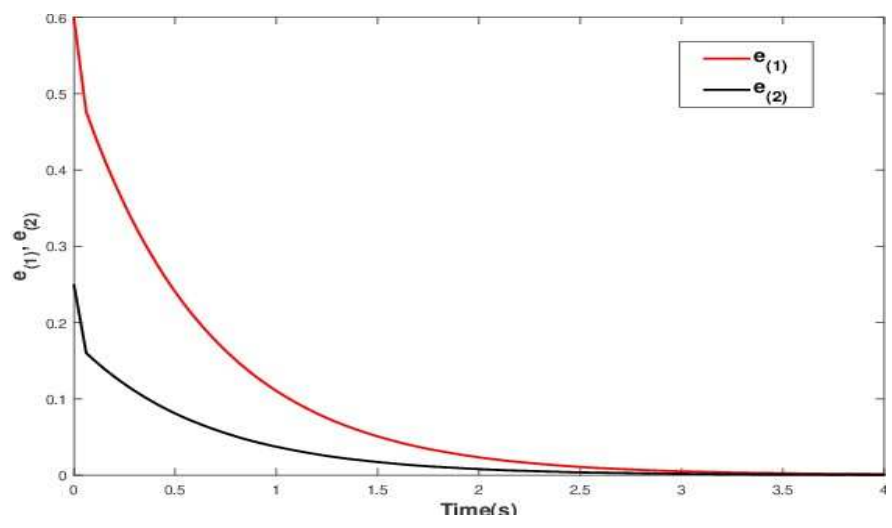

Fig. 1. Evolution of the tracking error dynamics starting at $e=[0.60 .25]^{T}$.

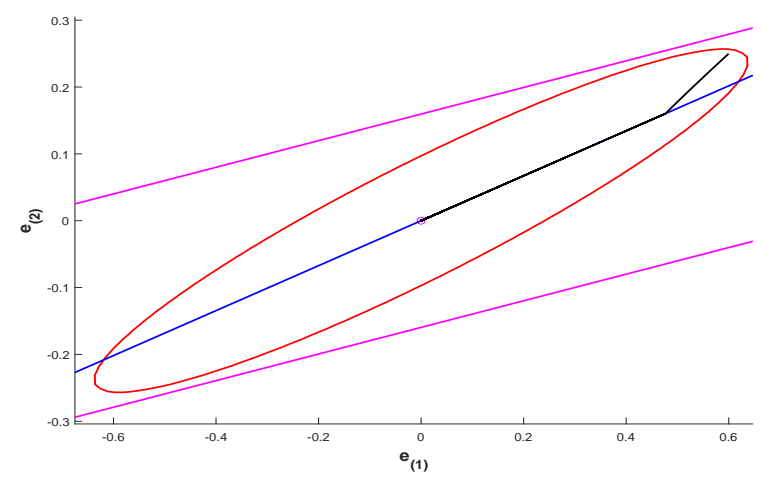

Fig. 2. Estimation of the domain of attraction (red line); trajectory of the tracking error (black line); Switching hyperplane (blue line).

\section{ILLUSTRATIVE EXAMPLE}

Consider the linear system (7) with $u \in \mathcal{V}=\{-v, v\}=$ $\{-1,1\}$, and matrices $A=\left[\begin{array}{cc}-1 & 0 \\ 0 & 0.1\end{array}\right]$ and $B=\left[\begin{array}{l}1 \\ 1\end{array}\right]$.

\section{A. Perfect tracking}

First we consider that the reference input $u_{r}$ takes values inside the set $\mathcal{V}^{r}=\left\{\frac{-v}{2}, \frac{v}{2}\right\}$ and is shown in Figure 3 . Considering a decay rate $\delta=1.5$, the set of LMIs (18)(19) are feasible for $Q=\left[\begin{array}{ll}0.4070 & 0.1519 \\ 0.1519 & 0.0661\end{array}\right]$ and $Y=$ $\left[\begin{array}{ll}-0.2221-0.1125\end{array}\right]$. Computer simulations of the tracking error evolution are performed for initial states $x(0)=$ $\left[\begin{array}{ll}0.6 & 0.25\end{array}\right]^{T}$ and $x_{r}(0)=\left[\begin{array}{ll}0 & 0\end{array}\right]^{T}$ and are reported in Figures 1-5.

As we can see from Figures 1 and 2, the tracking error trajectories starting in the domain of attraction $\mathcal{E}\left(Q^{-1}, 1\right)$ contained in the convex $\operatorname{Conv}\{\mathcal{V}\}$ (in red line) converge to the origin. From Figures 4 and 5 we can observe that the trajectory of the closed-loop system converge to the reference trajectory. These observations are consistent with the theoretical development in Section III

\section{B. Non-perfect tracking}

Here, we consider that the reference input $u_{r}$ takes values in the set $\mathcal{V}^{r}=\{-1,0,1\}$ and is shown in Figure 7. Considering a decay rate $\delta=0.5$, the LMI (25) is found feasible for 


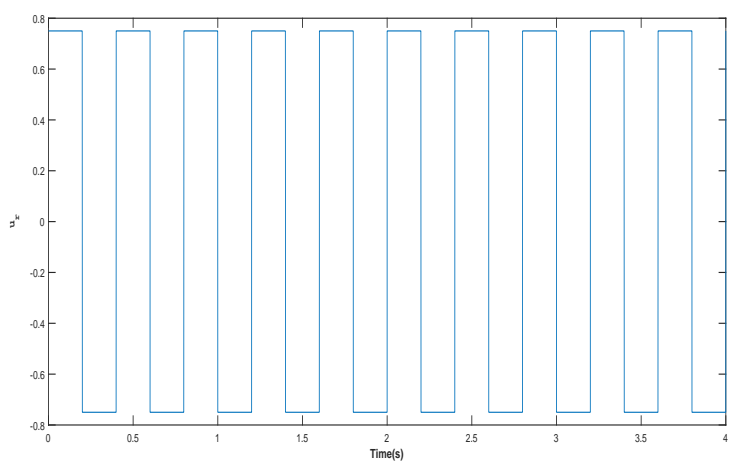

Fig. 3. Reference input $u_{r}$.

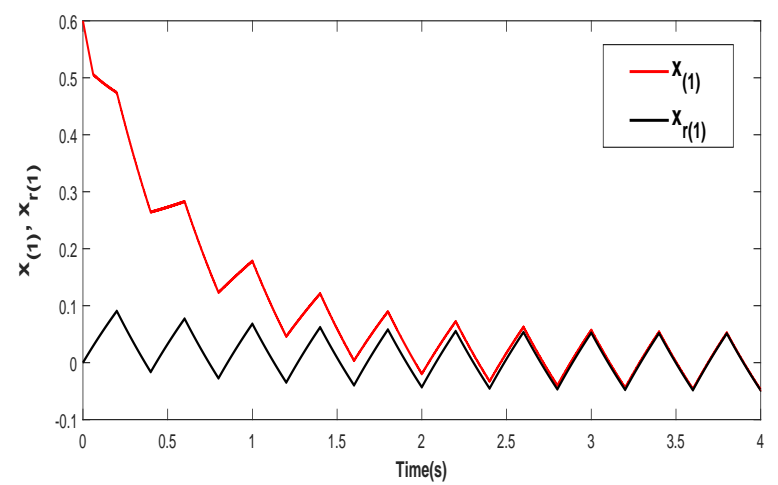

Fig. 4. Evolution of the state variables $x_{(1)}, x_{r(1)}$.

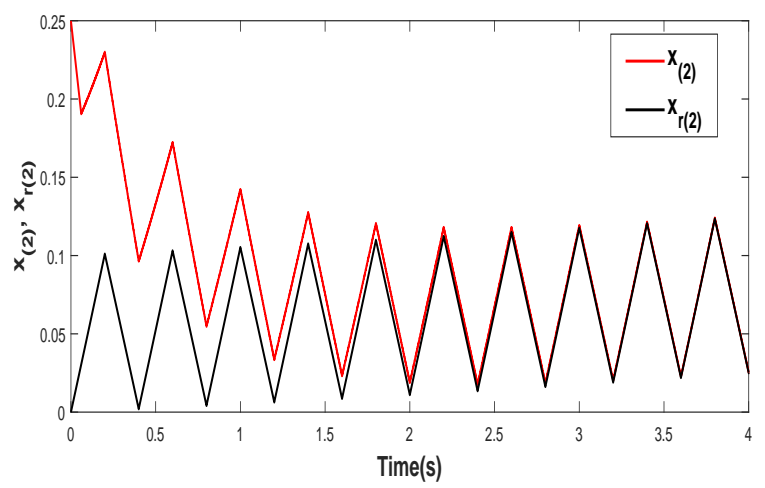

Fig. 5. Evolution of the state variables $x_{(2)}, x_{r(2)}$.

$Q=\left[\begin{array}{cc}1.6774 & -0.0251 \\ -0.0251 & 0.9877\end{array}\right]$ and $Y=\left[\begin{array}{ll}0.2744 & -0.6856\end{array}\right]$.

The comparison system (24) is such that $\xi_{\max }=2.0777$, $\eta(0)=1.5, \pi=3, \lambda=0.3074$ and $u_{r}$ is shown in Figure 6. Thus, under this conditions $\eta(t) \leq 5$ for all $t \geq 0$. Computer simulations of the tracking error evolution are performed for initial states $x(0)=\left[\begin{array}{ll}-1.53 & .02\end{array}\right]^{T}$ and $x_{r}(0)=\left[\begin{array}{ll}0 & 0\end{array}\right]^{T}$ and are reported in Figure 7-10.

From Figures 7 and 10 we can observe that the trajectories of the tracking errors initialized such that $V(e(0)) \leq 1.5$ remains bounded as $t$ tends to infinity. The state trajectories get and remain closer to the reference states as it can be seen

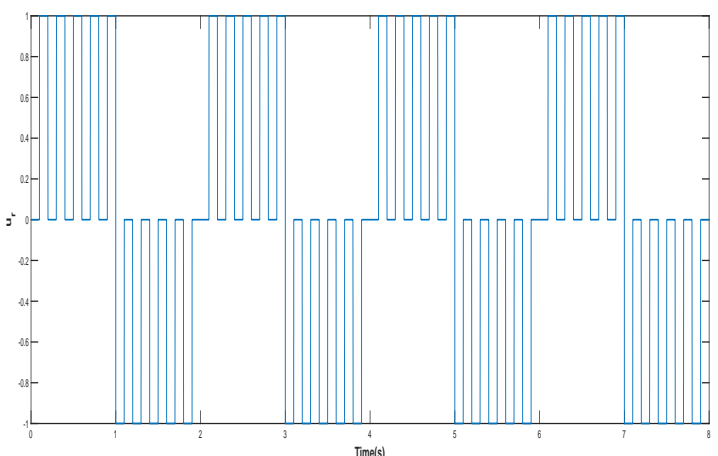

Fig. 6. Reference input $u_{r}$

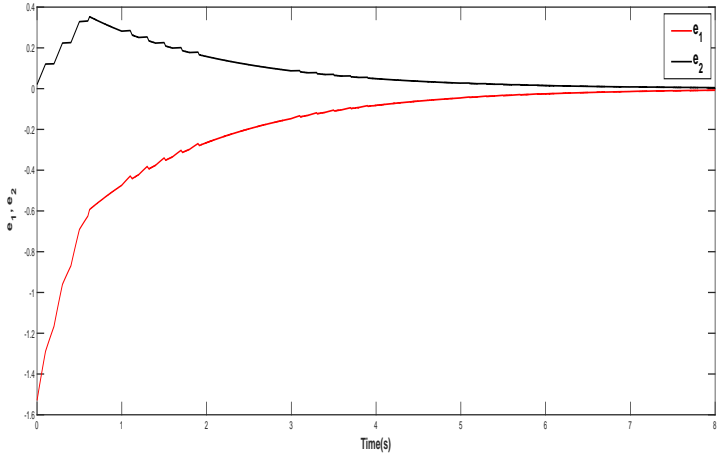

Fig. 7. Evolution of the tracking error dynamics starting at $e=[0.4-$ $0.06]^{T}$

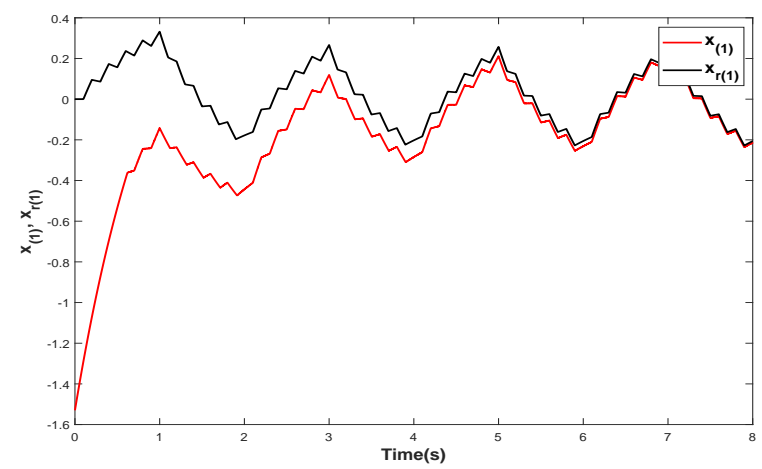

Fig. 8. Evolution of the state variables $x_{(1)}$ and $x_{r(1)}$

from Figures 8 and 9. These observations are consistent with the theoretical result shown in Section IV.

\section{CONClusion}

In this paper the problem of tracking a reference trajectory for Linear Time Invariant (LTI) systems using a relay feedback has been investigated. First, the problem of a reference tracking has been reformulated as a stabilization problem of the tracking error. Then, under some conditions on the reference inputs a design approach of a relay feedback controller that guarantees a perfect tracking of the reference 


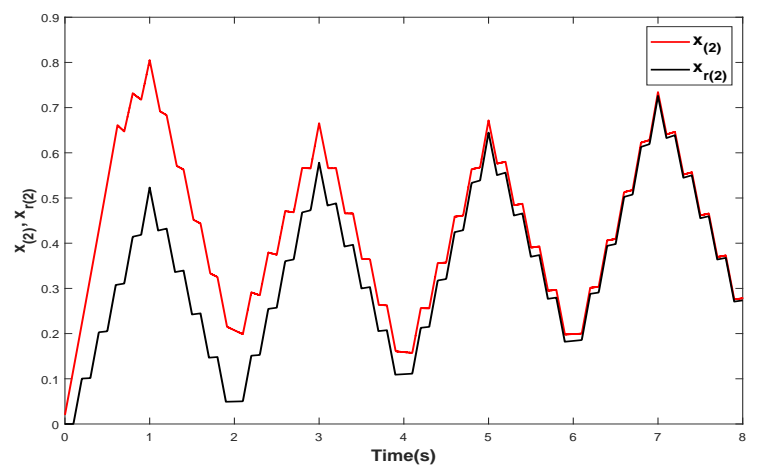

Fig. 9. Evolution of the state variables $x_{(2)}$ and $x_{r(2)}$

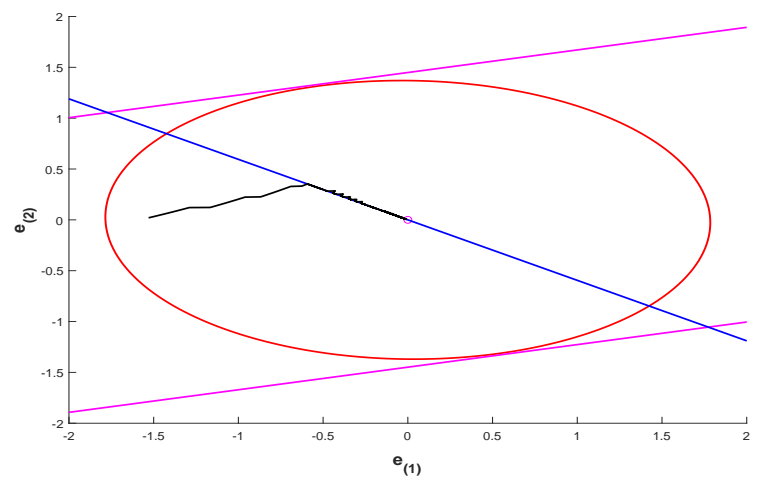

Fig. 10. Estimation of the domain of attraction (red line); trajectory of the tracking error (black line); Switching hyperplane (blue line).

trajectory together with an estimation of the domain of attraction has been provided. Moreover, conditions in LMI form have been proposed to compute a relay feedback and an estimation of the domain of attraction. Secondly, the case where the reference inputs can take the same values as the relay feedback has been considered. In this context, using the comparison principle, it has been shown that the designed relay feedback guarantees a practical stabilization of the tracking error. An estimation of the domain of attraction and the bound of the tracking error have been equally provided. Finally, computer simulations have been performed in order to show the efficiency of the developed method over a numerical example. In the future, the approach may be improved in various manners. For example, it is of interest to reduce the conservatism induced by Assumptions A-3 and A-4. In order to reduce the chattering, we can try to extend the approach to the case of min-switching strategy with mindwell time condition.

\section{APPENDIX I}

\section{PROOF OF THEOREM 1}

Proof: Thanks to the fact that the pair $(A, B)$ is stabilizable then there exists a gain $K$ such that $A_{c l}=$ $A+B K$ is Hurwitz. Furthermore, for all $\delta>0$ there exists a matrix $M=M^{T} \succ 0$ satisfying

$$
A_{c l}^{T} M+M A_{c l} \preceq-2 \delta M .
$$

Let us consider the quadratic Lyapunov function $V(e)=$ $e^{T} M e$. Our objective is to show that the differential inclusion (11) associated to the closed-loop system (7), (8) with $\mathrm{S}=$ $M B$, satisfy

$$
\sup _{y \in \mathcal{F}(t, e)} \frac{\partial V}{\partial e} y \leq-2 \alpha V(e),
$$

for some $\alpha>0$ in a domain $\mathcal{D} \subset \mathbb{R}^{n}$ that we will determine later on.

Now, let $\mathcal{I}^{*}(z)$ be the set of index for any $z \in \mathbb{R}^{n}$ such that

$$
\mathcal{I}^{*}(z)=\left\{i \in \mathcal{I}_{N}: z^{T} M B\left(v_{j}-v_{i}\right) \geq 0, \quad \forall j \in \mathcal{I}_{N}\right\} .
$$

To $\mathcal{I}^{*}(z)$ we associate for all $z \in \mathbb{R}^{n}$ the set $\psi^{*}(z)$ of vectors defined by

$$
\psi^{*}(z)=\left\{\beta \in \psi_{N}: \beta_{i}=0, \forall i \in \mathcal{I}_{N} \backslash \mathcal{I}^{*}(z)\right\} .
$$

Using (28) and (29), the set valued map $\mathcal{F}(t, e)$ in (12) satisfies

$$
\mathcal{F}(t, e) \subseteq \mathcal{F}^{*}(t, e)
$$

with

$$
\begin{aligned}
\mathcal{F}^{*}(t, e) & =\operatorname{Conv}_{i \in \mathcal{I}^{*}(e)}\left\{A e+B v_{i}-B u_{r}\right\} \\
& =\left\{A e+B v(\beta)-B u_{r}: \beta \in \psi^{*}(e)\right\},
\end{aligned}
$$

and $v(\beta)=\sum_{i=1}^{N} \beta_{i} v_{i}$.

Thus, in order to show (27), it is sufficient to prove that

$$
\sup _{y \in \mathcal{F}^{*}(t, e)} \frac{\partial V}{\partial e} y \leq-2 \alpha V(e),
$$

for some positive scalar $\alpha>0$ in some domain $\mathcal{D} \subset \mathbb{R}^{n}$ that will be determined later.

From (31), and thanks to the fact that $\psi^{*}(z)$ is compact for all $z \in \mathbb{R}^{n}$, we have

$$
\begin{aligned}
\sup _{y \in \mathcal{F}^{*}(t, e)} \frac{\partial V}{\partial e} y & =\sup _{\beta \in \psi^{*}(e)}\left\{\frac{\partial V}{\partial e}\left(A e+B v(\beta)-B u_{r}\right)\right\} \\
& =\max _{\beta \in \psi^{*}(e)}\left\{\frac{\partial V}{\partial e}\left(A e+B v(\beta)-B u_{r}\right)\right\} .
\end{aligned}
$$

Then, showing (32) is equivalent to demonstrating that for some $\alpha>0$

$$
\max _{\beta \in \psi^{*}(e)}\left\{\frac{\partial V}{\partial e}\left(A e+B v(\beta)-B u_{r}\right)\right\} \leq-2 \alpha V(e),
$$

in a domain $\mathcal{D} \subset \mathbb{R}^{n}$ to be determined below.

From inequality (26), we obtain

$$
\frac{\partial V}{\partial e}\left(A_{c l} e\right) \leq-2 \delta V(e), \forall e \in \mathbb{R}^{n} .
$$

Since $\operatorname{Conv}\left\{\mathcal{V}^{r}\right\} \subset \operatorname{int}\{\operatorname{Conv}\{\mathcal{V}\}\}$ then there exists $\varepsilon>0$ such that for all $c(e) \in \mathcal{B}(0, \varepsilon)$ and $v^{r} \in \mathcal{V}^{r}$ we have

$$
v^{r}+c(e) \in \operatorname{Conv}\{\mathcal{V}\} \text {. }
$$

Therefore, there exists a neighbourhood of the origin $\mathcal{E}(M, \gamma)$ with $\gamma>0$ such that for all $e \in \mathcal{E}(M, \gamma)$ we have

$$
K e \in \mathcal{B}(0, \varepsilon) \text {. }
$$


Thus, From (36) and (37), for all $e \in \mathcal{E}(M, \gamma)$ we have

$$
v^{r}+K e \in \operatorname{Conv}\{\mathcal{V}\} \text {. }
$$

Therefore, for all $e \in \mathcal{E}(M, \gamma)$ there exist positive scalars $\alpha_{j}(e), j \in \mathcal{I}_{N}$, such that $\sum_{j=1}^{N} \alpha_{j}(e)=1$ and

$$
v^{r}+K e=\sum_{j=1}^{N} \alpha_{j}(e) v_{j} .
$$

From now on, we consider the case where (35) and (39) are verified (i.e. for all $e \in \mathcal{E}(M, \gamma)$ ). From (28), for all $i \in \mathcal{I}^{*}(e)$ we have

$$
e^{T} M B\left(v_{j}-v_{i}\right) \geq 0, \forall j \in \mathcal{I}_{N}
$$

Then, for any $\beta \in \psi^{*}(e)$ we have

$$
e^{T} M B\left(v_{j}-v(\beta)\right) \geq 0, \forall j \in \mathcal{I}_{N} .
$$

Multiplying this last inequality by $\alpha_{j}(e)$ for $j \in \mathcal{I}_{N}\left(\alpha_{j}(e)\right.$ defined in (39)) and summing the $N$ elements, we obtain

$$
e^{T} M B\left(v^{r}+K e-v(\beta)\right) \geq 0,
$$

for all $v^{r} \in \mathcal{V}^{r}$.

Adding this to the left part of (34), for all $u_{r}=v^{r} \in \mathcal{V}^{r}$ and for all $e \in \mathcal{E}(M, \gamma)$ we get

$$
\begin{aligned}
& \max _{\beta \in \psi^{*}(e)}\left\{\frac{\partial V}{\partial e}\left(A e+B v(\beta)-B u_{r}\right)\right\} \\
& \leq e^{T}\left(A_{c l}^{T} M+M A_{c l}\right) e \\
& \leq-2 \delta V(e) .
\end{aligned}
$$

From the last inequality and taking $\alpha=\delta$, (34) is verified for all $u_{r}=v^{r} \in \mathcal{V}^{r}$ and for all $e \in \mathcal{E}(M, \gamma)$. Therefore, (26) is verified for all $u_{r}=v^{r} \in \mathcal{V}^{r}$ and for all $e \in \mathcal{E}(M, \gamma)$.

\section{APPENDIX II PROOF OF THEOREM 2}

Proof: We want to prove that if LMIs (18)-(19) are feasible then the closed-loop system (7), (8) is locally uniformly exponentially stable in some domain $\mathcal{D}$. Note that LMI (18) guarantees that the function $V(e)=e^{T} M e$ with $M=Q^{-1}$ satisfies

$$
\frac{\partial V}{\partial e}\left(A_{c l} e\right) \leq-2 \delta V(e), \forall e \in \mathbb{R}^{n} .
$$

with $A_{c l}=A+B K$ and $K=Y M$. Applying the Shur complement and taking into account the fact that $K=$ $Y Q^{-1}$, LMI (19) ensures that

$$
1-\tilde{h}_{i, j} K Q K^{T} \tilde{h}_{i, j}^{T} \geq 0, \forall i \in \mathcal{I}_{n_{h}}, \forall j \in \mathcal{I}_{\tilde{N}},
$$

with $\tilde{h}_{i, j}=\frac{h_{i}}{1-h_{i} v_{j}^{r}}$. Let us remark that the minimum of $V$ along the hyperplane $\tilde{h}_{i, j} K e=1$ is given by $\min _{\tilde{h}_{i, j} K e=1} e^{T} M e=\left(\tilde{h}_{i, j} K Q K^{T} \tilde{h}_{i, j}^{T}\right)^{-1}$. From (45) we have that for all $e \in \mathcal{E}(M, 1), \tilde{h}_{i, j} K e \leq 1, \forall i \in \mathcal{I}_{n_{h}}, \forall j \in \mathcal{I}_{\tilde{N}}$. By the definition of $\tilde{h}_{i, j}$, we obtain

$$
h_{i}\left(v_{j}^{r}+K e\right) \leq 1, \forall i \in \mathcal{I}_{n_{h}}, \forall j \in \mathcal{I}_{\tilde{N}} .
$$

Thus, the relation $u_{r}+K e \in \operatorname{Conv}\{\mathcal{V}\}$ holds. Therefore, from (46), (44) is satisfied for all $e \in \mathcal{E}(M, 1)$. From now on, using the same argument as in Theorem (1) one is able to show that

$$
\sup _{y \in \mathcal{F}(t, e)} \frac{\partial V}{\partial e} y \leq-2 \delta V(e), \forall e \in \mathcal{E}(M, 1) .
$$

\section{APPENDIX III PROOF OF THEOREM 3}

Proof: First, let us define for any $z \in \mathbb{R}^{n}$ the set of index $\mathcal{I}^{*}(z)$ such that

$$
\mathcal{I}^{*}(z)=\left\{i \in \mathcal{I}_{N}: z^{T} M B\left(v_{j}-v_{i}\right) \geq 0, \quad \forall j \in \mathcal{I}_{N}\right\} .
$$

To $\mathcal{I}^{*}(z)$ we associate for all $z \in \mathbb{R}^{n}$ the set $\psi^{*}(z)$ of vectors defined by

$$
\psi^{*}(z)=\left\{\beta \in \psi_{N}: \beta_{i}=0, \forall i \in \mathcal{I}_{N} \backslash \mathcal{I}^{*}(z)\right\} .
$$

Using (48) and (49), the set valued map $\mathcal{F}(t, e)$ in (12) satisfies

$$
\mathcal{F}(t, e) \subseteq \mathcal{F}^{*}(t, e)
$$

with

$$
\begin{aligned}
\mathcal{F}^{*}(t, e) & =\operatorname{Conv}_{i \in \mathcal{I}^{*}(e)}\left\{A e+B v_{i}-B u_{r}\right\} \\
& =\left\{A e+B v(\beta)-B u_{r}: \beta \in \psi^{*}(e)\right\},
\end{aligned}
$$

and $v(\beta)=\sum_{i=1}^{N} \beta_{i} v_{i}$. In order to show the result of Theorem 3, let us consider the quadratic Lyapunov function $V(e)=e^{T} M e$ where $M$ is defined in (21). Thus, From (21), one has

$$
\frac{\partial V}{\partial e}\left(A_{c l} e\right) \leq-2 \delta V(e), \forall e \in \mathbb{R}^{n} .
$$

Now, considering (50), let us evaluate the upper derivative $\dot{V}^{*}$ of the Lyapunov function along the solutions of the differential inclusion (11)

$$
\dot{V}^{*}(e)=\sup _{y \in \mathcal{F}^{*}(t, e)} \frac{\partial V}{\partial e} y .
$$

From (51), and using the fact that the set $\psi^{*}(z)$ is compact for all $z \in \mathbb{R}^{n}$, we have

$$
\begin{aligned}
\dot{V}^{*}(e) & =\sup _{\beta \in \psi^{*}(e)}\left\{\frac{\partial V}{\partial e}\left(A e+B v(\beta)-B u_{r}\right)\right\} \\
& =\max _{\beta \in \psi^{*}(e)}\left\{\frac{\partial V}{\partial e}\left(A e+B v(\beta)-B u_{r}\right)\right\} .
\end{aligned}
$$

From (48), for all $i \in \mathcal{I}^{*}(e)$ we have

$$
e^{T} M B\left(v_{j}-v_{i}\right) \geq 0, \forall j \in \mathcal{I}_{N} .
$$

Then, for any $\beta \in \psi^{*}(e)$ we have

$$
e^{T} M B\left(v_{j}-v(\beta)\right) \geq 0, \forall j \in \mathcal{I}_{N} .
$$

Multiplying this last inequality by $\alpha_{j}(e)$ for $j \in \mathcal{I}_{N}\left(\alpha_{j}(e)\right.$ defined in (23)) and summing the $N$ elements, we obtain

$$
e^{T} M B(K e-v(\beta)) \geq 0 .
$$


Adding this to the left part of (54), we get

$$
\dot{V}^{*}(e) \leq e^{T}\left(A_{c l}^{T} M+M A_{c l}\right) e-2 e^{T} M B u_{r} .
$$

Now, using (52), we obtain

$$
\dot{V}^{*}(e) \leq-2 \delta V(e)-2 e^{T} M B u_{r} .
$$

Recall that for any positive number $\theta$

$$
2 a^{T} b \leq \frac{1}{\theta} a^{T} a+\theta b^{T} b, \forall a, b \in \mathbb{R}^{n} .
$$

Applying (60) to the term $-2 e^{T} M B u_{r}$ with

$$
\theta=\pi, \quad a=\left(e^{T} M B\right)^{T}, \quad \text { and } \quad b=-u_{r},
$$

we obtain

$$
\begin{aligned}
\dot{V}^{*}(e) & \leq-2 \delta V(e)+\pi^{-1} e^{T} M B B^{T} M e \\
& +\pi u_{r}^{T} u_{r} .
\end{aligned}
$$

By the definition of $\xi_{\max }$ and $\lambda$ in (24), (62) becomes

$$
\begin{aligned}
\dot{V}^{*}(e) & \leq-\left(2 \delta-\pi^{-1} \xi_{\max }\right) V(e)+\pi u_{r}^{T} u_{r} \\
& =-\lambda V(e)+\pi u_{r}^{T} u_{r} .
\end{aligned}
$$

From Assumption A.4 and by the comparison principle [14] taking $V\left(e\left(t_{0}\right)\right) \leq \eta\left(t_{0}\right)$ i.e., $\left\|e\left(t_{0}\right)\right\| \leq R_{1}$ leads to

$$
V(e) \leq \eta(t) \leq \varepsilon \leq \gamma, \forall t \geq t_{0} \geq 0 .
$$

Thus,

$$
\|e(t)\| \leq R_{2}, \forall t \geq t_{0} \geq 0,
$$

which ends the proof.

\section{REFERENCES}

[1] J. P. Aubin and A. Cellina, Differential inclusions: set-valued maps and viability theory. Springer-Verlag New York, Inc., 1984.

[2] A. Bacciotti and L. Rosier, Liapunov functions and stability in control theory. Springer Science \& Business Media, 2006.

[3] I. Boiko, Discontinuous control systems: frequency-domain analysis and design. Springer Science \& Business Media, 2008.

[4] S. P. Boyd, L. El Ghaoui, E. Feron, and V. Balakrishnan, Linear matrix inequalities in system and control theory. SIAM, 1994, vol. 15.

[5] J. Cortes, "Discontinuous dynamical systems," Control Systems, vol. 28 , no. 3, pp. 36-73, 2008.

[6] C. Edwards and S. Spurgeon, Sliding mode control: theory and applications. Crc Press, 1998

[7] A. F. Filippov and F. M. Arscott, Differential equations with discontinuous righthand sides: control systems. Springer Science \& Business Media, 1988, vol. 18.

[8] J. M. Gonçalves, A. Megretski, M. Dahleh et al., "Global stability of relay feedback systems," Transactions on Automatic Control, vol. 46, no. 4 , pp. 550-562, 2001.

[9] L. Hetel, E. Fridman, and T. Floquet, "Variable structure control with generalized relays: A simple convex optimization approach," Transactions on Automatic Control, vol. 60, no. 2, pp. 497-502, 2015.

[10] K. H. Johansson, "Relay feedback and multivariable control," Ph.D. dissertation, Lund University, 1997.

[11] K. H. Johansson, A. E. Barabanov, and K. J. Åström, "Limit cycles with chattering in relay feedback systems," Transactions on Automatic Control, vol. 47, no. 9, pp. 1414-1423, 2002.

[12] K. H. Johansson, A. Rantzer et al., "Fast switches in relay feedback systems," Automatica, vol. 35, no. 4, pp. 539-552, 1999.

[13] Z. Kader, C. Fiter, L. Hetel, and L. Belkoura, "Stabilization by a relay control using non-quadratic lyapunov functions," Automatica, vol. 97, pp. 353-366, 2018.

[14] H. K. Khalil and J. W. Grizzle, Nonlinear systems. Prentice hall Upper Saddle River, N. J., 2002, vol. 3.
[15] D. Nesic and D. Liberzon, "A unified framework for design and analysis of networked and quantized control systems," Transactions on Automatic control, vol. 54, no. 4, pp. 732-747, 2009.

[16] A. Polyakov, "Practical stabilization via relay delayed control," in Conference on Decision and Control, 2008, pp. 5306-5311.

[17] _ _ " "On practical stabilization of systems with delayed relay control," Automation and Remote Control, vol. 71, no. 11, pp. 2331-2344, 2010.

[18] A. Poznyak, A. Polyakov, and V. Azhmyakov, Attractive Ellipsoids in Robust Control. Springer, 2014.

[19] Z. Retchkiman and G. Silva-Navarro, "Stabilization of nonlinear systems using vector lyapunov functions," IFAC Proceedings Volumes, vol. 31, no. 17, pp. 603-608, 1998.

[20] I. Z. TÍ iSypkin, Relay control systems. CUP Archive, 1984.

[21] V. Utkin, J. Guldner, and J. Shi, Sliding mode control in electromechanical systems. CRC press, 2009, vol. 34. 